\title{
EXISTENCE, COMPARISON, AND COMPACTNESS RESULTS FOR QUASILINEAR VARIATIONAL-HEMIVARIATIONAL INEQUALITIES
}

\author{
S. CARL, VY K. LE, AND D. MOTREANU \\ Received 31 May 2004 and in revised form 9 November 2004
}

We consider quasilinear elliptic variational-hemivariational inequalities involving the indicator function of some closed convex set and a locally Lipschitz functional. We provide a generalization of the fundamental notion of sub- and supersolutions, on the basis of which we then develop the sub-supersolution method for variational-hemivariational inequalities, including existence, comparison, compactness, and extremality results.

\section{Introduction}

Let $\Omega \subset \mathbb{R}^{N}$ be a bounded domain with Lipschitz boundary $\partial \Omega$, and let $V=W^{1, p}(\Omega)$ and $V_{0}=W_{0}^{1, p}(\Omega), 1<p<\infty$, denote the usual Sobolev spaces with their dual spaces $V^{*}$ and $V_{0}^{*}$, respectively. In this paper, we deal with the following quasilinear variationalhemivariational inequality:

$$
u \in K:\langle A u-f, v-u\rangle+\int_{\Omega} j^{o}(u ; v-u) d x \geq 0 \quad \forall v \in K,
$$

where $j^{o}(s ; r)$ denotes the generalized directional derivative of the locally Lipschitz function $j: \mathbb{R} \rightarrow \mathbb{R}$ at $s$ in the direction $r$ given by

$$
j^{o}(s ; r)=\limsup _{y \rightarrow s, t \downarrow 0} \frac{j(y+t r)-j(y)}{t},
$$

(cf., e.g., [6, Chapter 2]), $f \in V_{0}^{*}$, and $K$ is a closed and convex subset of $V_{0}$. The operator $A: V \rightarrow V_{0}^{*}$ is a second-order quasilinear differential operator in divergence form:

$$
A u(x)=-\sum_{i=1}^{N} \frac{\partial}{\partial x_{i}} a_{i}(x, \nabla u(x)) \quad \text { with } \nabla u=\left(\frac{\partial u}{\partial x_{1}}, \ldots, \frac{\partial u}{\partial x_{N}}\right) \text {. }
$$

The main goal of this paper is to develop the sub-supersolution method for variationalhemivariational inequalities of form (1.1). Problem (1.1) includes various special cases. 
(i) For $K=V_{0}$ and $j: \mathbb{R} \rightarrow \mathbb{R}$ smooth, (1.1) is the weak formulation of the Dirichlet problem

$$
u \in V_{0}: A u+j^{\prime}(u)=f \quad \text { in } V_{0}^{*},
$$

for which the sub-supersolution method is well known.

(ii) If $K=V_{0}$, and $j: \mathbb{R} \rightarrow \mathbb{R}$ not necessarily smooth, then (1.1) is a hemivariational inequality of the form

$$
u \in V_{0}:\langle A u-f, v-u\rangle+\int_{\Omega} j^{o}(u ; v-u) d x \geq 0 \quad \forall v \in V_{0},
$$

for which an extension of the sub-supersolution method has been given recently in [3].

(iii) If $j=0$, then (1.1) becomes a variational inequality for which a sub-supersolution method has been developed in $[8,9]$.

This paper continues the work on the extension of the sub-supersolution method started with the papers by Carl, Le, and Motreanu in [2, 3, 8, 9] to develop a strongly generalized and unified theory that includes all the above cited special cases.

\section{Notation and hypotheses}

We assume the following hypotheses of Leray-Lions type on the coefficient functions $a_{i}$, $i=1, \ldots, N$, of the operator $A$.

(A1) Each $a_{i}: \Omega \times \mathbb{R}^{N} \rightarrow \mathbb{R}$ satisfies the Carathéodory conditions, that is, $a_{i}(x, \xi)$ is measurable in $x \in \Omega$ for all $\xi \in \mathbb{R}^{N}$ and continuous in $\xi$ for almost all $x \in \Omega$. There exist a constant $c_{0}>0$ and a function $k_{0} \in L^{q}(\Omega), 1 / p+1 / q=1$, such that

$$
\left|a_{i}(x, \xi)\right| \leq k_{0}(x)+c_{0}|\xi|^{p-1}
$$

for a.e. $x \in \Omega$ and for all $\xi \in \mathbb{R}^{N}$.

(A2)

$$
\sum_{i=1}^{N}\left(a_{i}(x, \xi)-a_{i}\left(x, \xi^{\prime}\right)\right)\left(\xi_{i}-\xi_{i}^{\prime}\right)>0
$$

for a.e. $x \in \Omega$, and for all $\xi, \xi^{\prime} \in \mathbb{R}^{N}$ with $\xi \neq \xi^{\prime}$.

(A3)

$$
\sum_{i=1}^{N} a_{i}(x, \xi) \xi_{i} \geq \nu|\xi|^{p}-k_{1}(x)
$$

for a.e. $x \in \Omega$, and for all $\xi \in \mathbb{R}^{N}$ with some constant $\nu>0$ and some function $k_{1} \in L^{1}(\Omega)$. 
S. Carl et al. 403

As a consequence of (A1), (A2) the semilinear form $a$ associated with the operator $A$ by

$$
\langle A u, \varphi\rangle:=a(u, \varphi)=\int_{\Omega} \sum_{i=1}^{N} a_{i}(x, \nabla u) \frac{\partial \varphi}{\partial x_{i}} d x \quad \forall \varphi \in V_{0}
$$

is well defined for any $u \in V$, and the operator $A: V_{0} \rightarrow V_{0}^{*}$ is continuous, bounded, and strictly monotone. For functions $w, z: \Omega \rightarrow \mathbb{R}$ and sets $W$ and $Z$ of functions defined on $\Omega$ we use the following notations: $w \wedge z=\min \{w, z\}, w \vee z=\max \{w, z\}, W \wedge Z=\{w \wedge$ $z \mid w \in W, z \in Z\}, W \vee Z=\{w \vee z \mid w \in W, z \in Z\}$, and $w \wedge Z=\{w\} \wedge Z, w \vee Z=$ $\{w\} \vee Z$. Next we introduce our basic notion of sub-supersolution.

Definition 2.1. A function $\underline{u} \in V$ is called a subsolution of (1.1) if the following holds:

(i) $\underline{u} \leq 0$ on $\partial \Omega$,

(ii) $\langle A \underline{u}-f, v-\underline{u}\rangle+\int_{\Omega} j^{o}(\underline{u} ; v-\underline{u}) d x \geq 0$, for all $v \in \underline{u} \wedge K$.

Definition 2.2. $\bar{u} \in V$ is a supersolution of (1.1) if the following holds:

(i) $\bar{u} \geq 0$ on $\partial \Omega$,

(ii) $\langle A \bar{u}-f, v-\bar{u}\rangle+\int_{\Omega} j^{o}(\bar{u} ; v-\bar{u}) d x \geq 0$, for all $v \in \bar{u} \vee K$.

Note that the notion of sub-supersolution introduced here extends that for inclusions of hemivariational type introduced in $[4,5]$ and those for variational or hemivariational inequalities in $[3,8,9]$.

Let $\partial j: \mathbb{R} \rightarrow 2^{\mathbb{R}} \backslash\{\varnothing\}$ denote Clarke's generalized gradient of $j$ defined by

$$
\partial j(s):=\left\{\zeta \in \mathbb{R} \mid j^{o}(s ; r) \geq \zeta r, \forall r \in \mathbb{R}\right\} .
$$

We assume the following hypothesis for $j$.

(H) The function $j: \mathbb{R} \rightarrow \mathbb{R}$ is locally Lipschitz and its Clarke's generalized gradient $\partial j$ satisfies the following growth conditions:

(i) there exists a constant $c_{1} \geq 0$ such that

$$
\xi_{1} \leq \xi_{2}+c_{1}\left(s_{2}-s_{1}\right)^{p-1}
$$

for all $\xi_{i} \in \partial j\left(s_{i}\right), i=1,2$, and for all $s_{1}, s_{2}$ with $s_{1}<s_{2}$,

(ii) there is a constant $c_{2} \geq 0$ such that

$$
\xi \in \partial j(s):|\xi| \leq c_{2}\left(1+|s|^{p-1}\right) \quad \forall s \in \mathbb{R}
$$

Let $L^{p}(\Omega)$ be equipped with the natural partial ordering of functions defined by $u \leq w$ if and only if $w-u$ belongs to the positive cone $L_{+}^{p}(\Omega)$ of all nonnegative elements of $L^{p}(\Omega)$. This induces a corresponding partial ordering also in the subspace $V$ of $L^{p}(\Omega)$, and if $u, w \in V$ with $u \leq w$, then

$$
[u, w]=\{z \in V \mid u \leq z \leq w\}
$$

denotes the ordered interval formed by $u$ and $w$. 
In the proofs of our main results we make use of the cut-off function $b: \Omega \times \mathbb{R} \rightarrow \mathbb{R}$ related to an ordered pair of functions $\underline{u} \leq \bar{u}$, and given by

$$
b(x, s)= \begin{cases}(s-\bar{u}(x))^{p-1} & \text { if } s>\bar{u}(x), \\ 0 & \text { if } \underline{u}(x) \leq s \leq \bar{u}(x), \\ -(\underline{u}(x)-s)^{p-1} & \text { if } s<\underline{u}(x) .\end{cases}
$$

One readily verifies that $b$ is a Carathéodory function satisfying the growth condition

$$
|b(x, s)| \leq k(x)+c_{3}|s|^{p-1}
$$

for a.e. $x \in \Omega$, for all $s \in \mathbb{R}$, with some function $k \in L_{+}^{q}(\Omega)$ and a constant $c_{3} \geq 0$. Moreover, one has the following estimate

$$
\int_{\Omega} b(x, u(x)) u(x) d x \geq c_{4}\|u\|_{L^{p}(\Omega)}^{p}-c_{5} \quad \forall u \in L^{p}(\Omega)
$$

where $c_{4}$ and $c_{5}$ are some positive constants. In view of (2.10) the Nemytskij operator $B: L^{p}(\Omega) \rightarrow L^{q}(\Omega)$ defined by

$$
B u(x)=b(x, u(x))
$$

is continuous and bounded, and thus due to the compact embedding $V \subset L^{p}(\Omega)$ it follows that $B: V_{0} \rightarrow V_{0}^{*}$ is compact.

\section{Preliminaries}

In this section, we briefly recall a surjectivity result for multivalued mappings in reflexive Banach spaces (cf., e.g., [10, Theorem 2.12]) which among others will be used in the proof of our main result in this section.

Theorem 3.1. Let $X$ be a real reflexive Banach space with dual space $X^{*}, \Phi: X \rightarrow 2^{X^{*}}$ a maximal monotone operator, and $u_{0} \in \operatorname{dom}(\Phi)$. Let $A: X \rightarrow 2^{X^{*}}$ be a pseudomonotone operator, and assume that either $A_{u_{0}}$ is quasibounded or $\Phi_{u_{0}}$ is strongly quasibounded. Assume further that $A: X \rightarrow 2^{X^{*}}$ is $u_{0}$-coercive, that is, there exists a real-valued function $c: \mathbb{R}_{+} \rightarrow \mathbb{R}$ with $c(r) \rightarrow+\infty$ as $r \rightarrow+\infty$ such that for all $\left(u, u^{*}\right) \in \operatorname{graph}(A),\left\langle u^{*}, u-u_{0}\right\rangle \geq$ $c\left(\|u\|_{X}\right)\|u\|_{X}$ holds. Then $A+\Phi$ is surjective, that is, range $(A+\Phi)=X^{*}$.

The operators $A_{u_{0}}$ and $\Phi_{u_{0}}$ that appear in the theorem above are defined by $A_{u_{0}}(v):=$ $A\left(u_{0}+v\right)$ and similarly for $\Phi_{u_{0}}$. As for the notion of quasibounded and strongly quasibounded, we refer to [10, page 51]. In particular, one has that any bounded operator is quasibounded and strongly quasibounded as well. The following proposition provides sufficient conditions for an operator $A: X \rightarrow 2^{X^{*}}$ to be pseudomonotone, which is suitable for our purpose.

Proposition 3.2. Let $X$ be a real reflexive Banach space, and assume that $A: X \rightarrow 2^{X^{*}}$ satisfies the following conditions:

(i) for each $u \in X, A(u)$ is a nonempty, closed, and convex subset of $X^{*}$; 
(ii) $A: X \rightarrow 2^{X^{*}}$ is bounded;

(iii) if $u_{n} \rightarrow u$ in $X$ and $u_{n}^{*} \rightarrow u^{*}$ in $X^{*}$ with $u_{n}^{*} \in A\left(u_{n}\right)$ and if $\limsup \left\langle u_{n}^{*}, u_{n}-u\right\rangle \leq 0$, then $u^{*} \in A(u)$ and $\left\langle u_{n}^{*}, u_{n}\right\rangle \rightarrow\left\langle u^{*}, u\right\rangle$.

Then the operator $A: X \rightarrow 2^{X^{*}}$ is pseudomonotone.

As for the proof of Proposition 3.2 we refer, for example, to [10, Chapter 2].

\section{Existence and comparison result}

The main result of this section is given by the following theorem which provides an existence and comparison result for the variational-hemivariational inequality (1.1).

THeOREM 4.1. Let $\bar{u}$ and $\underline{u}$ be super- and subsolutions of (1.1), respectively, satisfying $\underline{u} \leq \bar{u}$, and assume $\bar{u} \wedge K \subset K$ and $\underline{u} \vee K \subset K$. Then under hypotheses (A1)-(A3) and (H), there exist solutions of (1.1) within the ordered interval $[\underline{u}, \bar{u}]$.

Proof. Let $I_{K}: V_{0} \rightarrow \mathbb{R} \cup\{+\infty\}$ denote the indicator function related to the given closed convex set $K \neq \varnothing$ and defined by

$$
I_{K}(u)= \begin{cases}0 & \text { if } u \in K, \\ +\infty & \text { if } u \notin K\end{cases}
$$

which is proper, convex, and lower semicontinuous. By means of the indicator function the variational-hemivariational inequality (1.1) can be rewritten in the following form. Find $u \in K$ such that

$$
\langle A u-f, v-u\rangle+I_{K}(v)-I_{K}(u)+\int_{\Omega} j^{o}(u ; v-u) d x \geq 0 \quad \forall v \in V_{0} .
$$

Since we are looking for solutions of (4.2) within $[\underline{u}, \bar{u}]$, we consider the following auxiliary problem: Find $u \in K$ such that

$$
\langle A u-f+\lambda B(u), v-u\rangle+I_{K}(v)-I_{K}(u)+\int_{\Omega} j^{o}(u ; v-u) d x \geq 0 \quad \forall v \in V_{0},
$$

where $B$ is the cut-off operator introduced in Section 2, and $\lambda \geq 0$ is some parameter to be specified later. As will be seen in the course of the proof, the role of $\lambda B$ is twofold. First it provides a coercivity generating term, and second, it allows for comparison. The proof of the theorem will be done in two steps. In Step 1 we prove the existence of solutions of auxiliary problem (4.3), and in Step 2 we are going to show that any solution of (4.3) belongs to the interval $[\underline{u}, \bar{u}]$, which completes the proof, since then $B(u)=0$ and $(4.2)$ holds.

Step 1 (existence for (4.3)). We introduce the functional $J: L^{p}(\Omega) \rightarrow \mathbb{R}$ defined by

$$
J(v)=\int_{\Omega} j(v(x)) d x \quad \forall v \in L^{p}(\Omega),
$$


which by hypothesis $(\mathrm{H})$ is locally Lipschitz, and moreover, by Aubin-Clarke theorem (see [6, page 83]) for each $u \in L^{p}(\Omega)$ we have

$$
\xi \in \partial J(u) \Longrightarrow \xi \in L^{q}(\Omega) \quad \text { with } \xi(x) \in \partial j(u(x)) \text { for a.e. } x \in \Omega \text {. }
$$

Consider now the multivalued operator

$$
A+\lambda B+\partial\left(\left.J\right|_{V_{0}}\right)+\partial I_{K}: V_{0} \longrightarrow 2^{V_{0}^{*}}
$$

where $\left.J\right|_{V_{0}}$ denotes the restriction of $J$ to $V_{0}$ and $\partial I_{K}$ is the subdifferential of $I_{K}$ in the sense of convex analysis. It is well known that $\Phi:=\partial I_{K}: V_{0} \rightarrow 2^{V_{0}^{*}}$ is a maximal monotone operator (cf., e.g., [11]). Since $A: V_{0} \rightarrow V_{0}^{*}$ is strictly monotone, bounded, and continuous, and $\lambda B: V_{0} \rightarrow V_{0}^{*}$ is bounded, continuous, and compact, it follows that $A+\lambda B: V_{0} \rightarrow V_{0}^{*}$ is a (single-valued) pseudomonotone, continuous, and bounded operator. In [3], it has been shown that $\partial\left(\left.J\right|_{V_{0}}\right): V_{0} \rightarrow 2^{V_{0}^{*}}$ is a (multivalued) pseudomonotone operator, which, due to $(\mathrm{H})$, is bounded. Thus $A_{0}:=A+\lambda B+\partial\left(\left.J\right|_{V_{0}}\right): V_{0} \rightarrow 2^{V_{0}^{*}}$ is a pseudomonotone and bounded operator. Hence, it follows by Theorem 3.1 that range $\left(A_{0}+\Phi\right)=V_{0}^{*}$ provided $A_{0}$ is $u_{0}$-coercive for some $u_{0} \in K$, which can readily be seen as follows. For any $v \in V_{0}$ and any $w \in \partial\left(\left.J\right|_{V_{0}}\right)(v)$, we obtain by applying (A3), (H)(ii), and (2.11) the estimate

$$
\begin{aligned}
\langle A v+ & \left.\lambda B(v)+w, v-u_{0}\right\rangle \\
= & \int_{\Omega} \sum_{i=1}^{N} a_{i}(x, \nabla v) \frac{\partial v}{\partial x_{i}} d x+\lambda\langle B(v), v\rangle+\int_{\Omega} w v d x-\left\langle A v+\lambda B(v)+w, u_{0}\right\rangle \\
\geq & v \int_{\Omega}|\nabla v|^{p} d x-\left\|k_{1}\right\|_{L^{1}(\Omega)}+c_{4} \lambda\|v\|_{L^{p}(\Omega)}^{p}-c_{5} \lambda \\
& -c_{2} \int_{\Omega}\left(1+|v|^{p-1}\right)|v| d x-\left|\left\langle A v+\lambda B(v)+w, u_{0}\right\rangle\right| \\
\geq & v\|v\|_{V_{0}}^{p}-C\left(1+\|v\|_{V_{0}}^{p-1}\right)
\end{aligned}
$$

for some constant $C>0$, by choosing the constant $\lambda$ in such a way that $c_{4} \lambda>c_{2}$. Since $p>1$, the coercivity of $A_{0}$ follows from (4.7). In view of the surjectivity of the operator $A_{0}+\Phi$, there exists a $u \in K$ such that $f \in A_{0}(u)+\Phi(u)$, that is, there is an $\xi \in \partial\left(\left.J\right|_{V_{0}}\right)(u)$ with $\xi \in L^{q}(\Omega)$ and $\xi(x) \in \partial j(u(x))$ for a.e. $x \in \Omega$, and an $\eta \in \Phi(u)$ such that

$$
A u-f+\lambda B(u)+\xi+\eta=0 \quad \text { in } V_{0}^{*},
$$

where

$$
\begin{array}{cc}
\langle\xi, \varphi\rangle=\int_{\Omega} \xi(x) \varphi(x) d x & \forall \varphi \in V_{0}, \\
I_{K}(v) \geq I_{K}(u)+\langle\eta, v-u\rangle & \forall v \in V_{0} .
\end{array}
$$

By definition of Clarke's generalized gradient $\partial j$ from (4.9) we get

$$
\langle\xi, \varphi\rangle=\int_{\Omega} \xi(x) \varphi(x) d x \leq \int_{\Omega} j^{o}(u(x) ; \varphi(x)) d x \quad \forall \varphi \in V_{0} .
$$


Thus from (4.8), (4.9), (4.10), and (4.11) with $\varphi$ replaced by $v-u$ we obtain (4.3), which proves the existence of solutions of problem (4.3).

Step $2(\underline{u} \leq u \leq \bar{u}$ for any solution $u$ of (4.3)). We first show $u \leq \bar{u}$. By definition, the supersolution $\bar{u}$ satisfies $\bar{u} \geq 0$ on $\partial \Omega$, and

$$
\langle A \bar{u}-f, v-\bar{u}\rangle+\int_{\Omega} j^{o}(\bar{u} ; v-\bar{u}) d x \geq 0 \quad \forall v \in \bar{u} \vee K .
$$

Let $u$ be any solution of (4.3) which is equivalent to the following variational-hemivariational inequality:

$$
u \in K:\langle A u-f, v-u\rangle+\langle\lambda B(u), v-u\rangle+\int_{\Omega} j^{o}(u ; v-u) d x \geq 0 \quad \forall v \in K .
$$

We apply the special test function $v=\bar{u} \vee u=\bar{u}+(u-\bar{u})^{+}(\in \bar{u} \vee K)$ in (4.12) and $v=\bar{u} \wedge$ $u=u-(u-\bar{u})^{+}(\in K)$ in (4.13), and get by adding the resulting inequalities the following one:

$$
\begin{aligned}
& \left\langle A \bar{u}-A u,(u-\bar{u})^{+}\right\rangle+\lambda\left\langle B(u),-(u-\bar{u})^{+}\right\rangle \\
& \quad+\int_{\Omega}\left(j^{o}\left(\bar{u} ;(u-\bar{u})^{+}\right)+j^{o}\left(u ;-(u-\bar{u})^{+}\right)\right) d x \geq 0,
\end{aligned}
$$

which yields due to

$$
\left\langle A u-A \bar{u},(u-\bar{u})^{+}\right\rangle \geq 0
$$

the inequality

$$
\lambda\left\langle B(u),(u-\bar{u})^{+}\right\rangle \leq \int_{\Omega}\left(j^{o}\left(\bar{u} ;(u-\bar{u})^{+}\right)+j^{o}\left(u ;-(u-\bar{u})^{+}\right)\right) d x .
$$

By using $(\mathrm{H})$ and the properties on $j^{o}$ and $\partial j$ we get for certain $\bar{\xi}(x) \in \partial j(\bar{u}(x))$ and $\xi(x) \in \partial j(u(x))$ the following estimate of the right-hand side of (4.16):

$$
\begin{aligned}
\int_{\Omega}\left(j^{o}\left(\bar{u} ;(u-\bar{u})^{+}\right)+j^{o}\left(u ;-(u-\bar{u})^{+}\right)\right) d x \\
\quad=\int_{\{u>\bar{u}\}}\left(j^{o}(\bar{u} ; u-\bar{u})+j^{o}(u ;-(u-\bar{u}))\right) d x \\
\quad=\int_{\{u>\bar{u}\}}(\bar{\xi}(x)(u(x)-\bar{u}(x))+\xi(x)(-(u(x)-\bar{u}(x)))) d x \\
\quad=\int_{\{u>\bar{u}\}}(\bar{\xi}(x)-\xi(x))(u(x)-\bar{u}(x)) d x \\
\leq \int_{\{u>\bar{u}\}} c_{1}(u(x)-\bar{u}(x))^{p} d x .
\end{aligned}
$$

Since

$$
\left\langle B(u),(u-\bar{u})^{+}\right\rangle=\int_{\{u>\bar{u}\}}(u-\bar{u})^{p} d x,
$$


we get from (4.16) and (4.17) the estimate

$$
\left(\lambda-c_{1}\right) \int_{\{u>\bar{u}\}}(u-\bar{u})^{p} d x \leq 0 .
$$

Selecting the parameter $\lambda$, in addition, such that $\lambda-c_{1}>0$, then (4.19) yields

$$
\int_{\Omega}\left((u-\bar{u})^{+}\right)^{p} d x \leq 0
$$

which implies $(u-\bar{u})^{+}=0$ and thus $u \leq \bar{u}$. The proof for the inequality $\underline{u} \leq u$ can be carried out in a similar way which completes the proof of the theorem.

\section{Compactness and existence of extremal solutions}

Let $\mathscr{Y}$ denote the set of all solutions of $(1.1)$ within the interval $[\underline{u}, \bar{u}]$ of an ordered pair of sub- and supersolutions. In this section, we are going to show that the solution set $\mathscr{Y}$ is compact, and under certain lattice conditions on $K, \mathscr{Y}$ possesses the smallest and greatest elements with respect to the given partial ordering. The smallest and greatest elements of $\mathscr{S}$ are called the extremal solutions of (1.1) within $[\underline{u}, \bar{u}]$.

Theorem 5.1. Under the hypotheses of Theorem 4.1 the solution set $\mathscr{Y}$ is compact in $V_{0}$.

Proof. First we prove that $\mathscr{S}$ is bounded in $V_{0}$. Since any $u \in \mathscr{S}$ belongs to the interval $[\underline{u}, \bar{u}]$, it follows that $\mathscr{Y}$ is bounded in $L^{p}(\Omega)$. Moreover, any $u \in \mathscr{Y}$ solves (1.1), that is, we have $u \in K:\langle A u-f, v-u\rangle+\int_{\Omega} j^{o}(u ; v-u) d x \geq 0$, for all $v \in K$. Let $u_{0}$ be any (fixed) element of $K$. By taking $v=u_{0}$ in the above inequality we get

$$
\langle A u, u\rangle \leq\left\langle A u, u_{0}\right\rangle+\left\langle f, u-u_{0}\right\rangle+\int_{\Omega} j^{o}\left(u ; u_{0}-u\right) d x .
$$

This yields, by applying (A3), (H)(ii), and Young's inequality, the following estimate:

$$
\nu\|\nabla u\|_{L^{p}(\Omega)}^{p} \leq\left\|k_{1}\right\|_{L^{1}(\Omega)}+c(\varepsilon)\left(\|f\|_{V_{0}^{*}}^{q}+1\right)+\varepsilon\|u\|_{V_{0}}^{p}+\tilde{\alpha}\left(\|u\|_{L^{p}(\Omega)}+\|u\|_{L^{p}(\Omega)}^{p}+1\right)
$$

for any $\varepsilon>0$. Hence, the boundedness of $\mathscr{S}$ in $V_{0}$ follows by choosing $\varepsilon$ sufficiently small and by taking into account that $\mathscr{Y}$ is bounded in $L^{p}(\Omega)$.

Let $\left(u_{n}\right) \subset \mathscr{Y}$. From the above boundedness of $\mathscr{Y}$ in $V_{0}$, we can choose a subsequence $\left(u_{k}\right)$ of $\left(u_{n}\right)$ such that

$$
u_{k} \rightarrow u \quad \text { in } V_{0}, \quad u_{k} \longrightarrow u \quad \text { in } L^{p}(\Omega), \quad u_{k}(x) \longrightarrow u(x) \quad \text { a.e. in } \Omega \text {. }
$$

Obviously $u \in[\underline{u}, \bar{u}]$. On the other hand, because $K$ is closed and convex in $V_{0}$, it is weakly closed. As $u_{k} \in K$ for all $k$, we see that $u$ is also in $K$. Since $u_{k}$ solve (1.1), we can put $v=u \in K$ in (1.1) (with $u_{k}$ instead of $u$ ) and get

$$
\left\langle A u_{k}-f, u-u_{k}\right\rangle+\int_{\Omega} j^{o}\left(u_{k} ; u-u_{k}\right) d x \geq 0,
$$


and thus

$$
\left\langle A u_{k}, u_{k}-u\right\rangle \leq\left\langle f, u_{k}-u\right\rangle+\int_{\Omega} j^{o}\left(u_{k} ; u-u_{k}\right) d x .
$$

Due to (5.3) and due to the fact that $(s, r) \mapsto j^{o}(s ; r)$ is upper semicontinuous, we get by applying Fatou's lemma

$$
\underset{k}{\limsup } \int_{\Omega} j^{o}\left(u_{k} ; u-u_{k}\right) d x \leq \int_{\Omega} \limsup _{k} j^{o}\left(u_{k} ; u-u_{k}\right) d x=0 .
$$

In view of (5.6) we thus obtain from (5.3) and (5.5)

$$
\limsup _{k}\left\langle A u_{k}, u_{k}-u\right\rangle \leq 0 .
$$

Since the operator $A$ has the $\left(S_{+}\right)$-property (we refer, e.g., to [1] for the definition of the $\left(\mathrm{S}_{+}\right)$-property being used here), the weak convergence of $\left(u_{k}\right)$ in $V_{0}$ along with (5.7) imply the strong convergence $u_{k} \rightarrow u$ in $V_{0}$, see, for example, [1, Theorem D.2.1]. Moreover, the limit $u$ belongs to $\mathscr{S}$ as can be seen by passing to the limsup on the left-hand side of the following inequality:

$$
\left\langle A u_{k}-f, v-u_{k}\right\rangle+\int_{\Omega} j^{o}\left(u_{k} ; v-u_{k}\right) d x \geq 0,
$$

where we have used Fatou's lemma and the strong convergence of $\left(u_{k}\right)$ in $V_{0}$. This completes the proof.

As for the existence of extremal solutions in $\mathscr{Y}$, we introduce the following notion.

Definition 5.2. Let $(\mathscr{P}, \leq)$ be a partially ordered set. A subset $\mathscr{C}$ of $\mathscr{P}$ is said to be upwarddirected if for each pair $x, y \in \mathscr{C}$, there is a $z \in \mathscr{C}$ such that $x \leq z$ and $y \leq z$, and $\mathscr{C}$ is downward-directed if for each pair $x, y \in \mathscr{C}$, there is a $w \in \mathscr{C}$ such that $w \leq x$ and $w \leq y$. If $\mathscr{C}$ is both upward and downward directed it is called directed.

We are now ready to prove our extremality result.

Theorem 5.3. Let the hypotheses of Theorem 4.1 be satisfied, and assume, moreover,

$$
K \wedge K \subset K, \quad K \vee K \subset K
$$

Then, the solution set 9 possesses extremal elements.

Proof. The proof of Theorem 5.3 is divided into two steps. In Step 1, we show that the solution set $\mathscr{Y}$ is directed, and the existence of extremal elements of $\mathscr{Y}$ is proved in Step 2.

Step 1 ( $\mathscr{S}$ is a directed set). As a consequence of Theorem 4.1 , we have $\mathscr{S} \neq \varnothing$. Given $u_{1}, u_{2} \in \mathscr{Y}$, we show that there is a $u \in \mathscr{S}$ such that $u_{k} \leq u, k=1,2$, which means $\mathscr{S}$ is upward-directed. To this end we consider the following auxiliary variational-hemivariational inequality

$$
u \in K:\langle A u-f+\lambda B(u), v-u\rangle+\int_{\Omega} j^{o}(u ; v-u) d x \geq 0 \quad \forall v \in K,
$$


where $\lambda \geq 0$ is a free parameter to be chosen later. Unlike in the proof of Theorem 4.1, the operator $B$ is now given by the following cut-off function $b: \Omega \times \mathbb{R} \rightarrow \mathbb{R}$ :

$$
b(x, s)= \begin{cases}(s-\bar{u}(x))^{p-1} & \text { if } s>\bar{u}(x) \\ 0 & \text { if } u_{0}(x) \leq s \leq \bar{u}(x) \\ -\left(u_{0}(x)-s\right)^{p-1} & \text { if } s<u_{0}(x)\end{cases}
$$

where $u_{0}=\max \left\{u_{1}, u_{2}\right\}$. By arguments similar to those in the proof of Theorem 4.1 we get the existence of solutions of (5.10). The set $\mathscr{Y}$ is shown to be upward-directed provided that any solution $u$ of (5.10) satisfies $u_{k} \leq u \leq \bar{u}, k=1,2$, because then $B u=0$ and thus $u \in \mathscr{S}$ exceeding $u_{k}$.

For $k=1,2$, because $u_{k} \in \mathscr{Y}$, we have $u_{k} \in K \cap[\underline{u}, \bar{u}]$ and

$$
\left\langle A u_{k}-f, v-u_{k}\right\rangle+\int_{\Omega} j^{o}\left(u_{k} ; v-u_{k}\right) d x \geq 0 \quad \forall v \in K
$$

Note that since $u, u_{1}, u_{2} \in K,(5.9)$ implies that

$$
u+\left(u_{k}-u\right)^{+}=u \vee u_{k} \in K, \quad u_{k}-\left(u_{k}-u\right)^{+}=u \wedge u_{k} \in K
$$

Therefore, one can take as special functions $v=u+\left(u_{k}-u\right)^{+}$in $(5.10)$ and $v=u_{k}-\left(u_{k}-\right.$ $u)^{+}$in (5.12). Adding the resulting inequalities we obtain

$$
\begin{aligned}
\left\langle A u_{k}\right. & \left.-A u,\left(u_{k}-u\right)^{+}\right\rangle-\lambda\left\langle B(u),\left(u_{k}-u\right)^{+}\right\rangle \\
& \leq \int_{\Omega}\left(j^{o}\left(u ;\left(u_{k}-u\right)^{+}\right)+j^{o}\left(u_{k} ;-\left(u_{k}-u\right)^{+}\right)\right) d x .
\end{aligned}
$$

Arguing as in (4.17), we have for the right-hand side of (5.14) the estimate

$$
\int_{\Omega}\left(j^{o}\left(u ;\left(u_{k}-u\right)^{+}\right)+j^{o}\left(u_{k} ;-\left(u_{k}-u\right)^{+}\right)\right) d x \leq \int_{\left\{u_{k}>u\right\}} c_{1}\left(u_{k}(x)-u(x)\right)^{p} d x .
$$

For the terms on the left-hand side we have

$$
\left\langle A u_{k}-A u,\left(u_{k}-u\right)^{+}\right\rangle \geq 0
$$

and (5.11) yields

$$
\begin{aligned}
\left\langle B(u),\left(u_{k}-u\right)^{+}\right\rangle & =-\int_{\left\{u_{k}>u\right\}}\left(u_{0}(x)-u(x)\right)^{p-1}\left(u_{k}(x)-u(x)\right) d x \\
& \leq-\int_{\left\{u_{k}>u\right\}}\left(u_{k}(x)-u(x)\right)^{p} d x .
\end{aligned}
$$

By means of (5.15), (5.16), (5.17) we get from (5.14) the inequality

$$
\left(\lambda-c_{1}\right) \int_{\left\{u_{k}>u\right\}}\left(u_{k}(x)-u(x)\right)^{p} d x \leq 0 .
$$


Selecting $\lambda$ such that $\lambda>c_{1}$ from (5.18) it follows $u_{k} \leq u$. The proof for $u \leq \bar{u}$ follows similar arguments, and thus $\mathscr{S}$ is upward-directed. By obvious modifications of the auxiliary problem, one can show analogously that $\mathscr{S}$ is also downward-directed.

Step 2 (existence of extremal solutions). We show the existence of the greatest element of $\mathscr{Y}$. Since $V_{0}$ is separable, we have that $\mathscr{S} \subset V_{0}$ is separable too, so there exists a countable, dense subset $Z=\left\{z_{n} \mid n \in \mathbb{N}\right\}$ of $\mathscr{Y}$. From Step 1, $\mathscr{S}$ is upward-directed, so we can construct an increasing sequence $\left(u_{n}\right) \subset \mathscr{S}$ as follows. Let $u_{1}=z_{1}$. Select $u_{n+1} \in \mathscr{S}$ such that

$$
\max \left\{z_{n}, u_{n}\right\} \leq u_{n+1} \leq \bar{u} .
$$

The existence of $u_{n+1}$ is established in Step 1. From the compactness of $\mathscr{S}$ according to Theorem 5.1, we can choose a subsequence of $\left(u_{n}\right)$, denoted again $\left(u_{n}\right)$, and an element $u \in \mathscr{S}$ such that $u_{n} \rightarrow u$ in $V_{0}$, and $u_{n}(x) \rightarrow u(x)$ a.e. in $\Omega$. This last property of $\left(u_{n}\right)$ combined with its increasing monotonicity implies that the entire sequence is convergent in $V_{0}$ and, moreover, $u=\sup _{n} u_{n}$. By construction, we see that

$$
\max \left\{z_{1}, z_{2}, \ldots, z_{n}\right\} \leq u_{n+1} \leq u \quad \forall n,
$$

thus $Z \subset[\underline{u}, u]$. Since the interval $[\underline{u}, u]$ is closed in $V_{0}$, we infer

$$
\mathscr{S} \subset \bar{Z} \subset \overline{[\underline{u}, u]}=[\underline{u}, u],
$$

which in conjunction with $u \in \mathscr{S}$ ensures that $u$ is the greatest solution of (1.1).

The existence of the least solution of (1.1) can be proved in a similar way.

Remark 5.4. From the proof of Theorem 5.3 it can be seen that instead of lattice condition (5.9), it is enough to assume the following weaker condition:

$$
K \wedge(K \cap[\underline{u}, \bar{u}]) \subset K, \quad K \vee(K \cap[\underline{u}, \bar{u}]) \subset K .
$$

\section{Example and generalization}

6.1. Example. We consider (1.1) with $f \in L^{p^{* \prime}}(\Omega)$, where $p^{*^{\prime}}$ is the Hölder conjugate of the critical Sobolev exponent $p^{*}$, and $K$ representing the following obstacle problem:

$$
K=\left\{v \in V_{0} \mid v(x) \leq \psi(x) \text { for a.e. } x \in \Omega\right\}
$$

with $\psi: \Omega \rightarrow \mathbb{R}$ measurable. We are going to provide sufficient conditions for the existence of an ordered pair of constant sub-and supersolutions $\alpha$ and $\beta$, respectively.

Proposition 6.1. Let $K \neq \varnothing$ be given by (6.1) and assume $f$ and $\psi$ as given above, and let $a_{i}(x, 0)=0, i=1, \ldots, N$. Then

(a) the constant function $\underline{u}(x) \equiv \alpha \leq 0$ is a subsolution of (1.1) if

$$
f(x) \geq-j^{o}(\alpha ;-1) \quad \text { for a.e. } x \in \Omega,
$$

(b) the constant function $\bar{u}(x) \equiv \beta \geq 0$ is a supersolution of (1.1) if

$$
f(x) \leq j^{o}(\beta ; 1) \text { for a.e. } x \in \Omega \text {, }
$$


(c) if $f \in L^{\infty}(\Omega)$ and $\alpha, \beta \in \mathbb{R}$ satisfy $\alpha \leq 0 \leq \beta$ and

$$
-j^{o}(\alpha ;-1) \leq f(x) \leq j^{o}(\beta ; 1) \quad \text { for a.e. } x \in \Omega,
$$

then $\alpha$ and $\beta$ is an ordered pair of sub-and supersolutions.

Proof. Let $\alpha \leq 0$ satisfy (6.2). According to Definition 2.1 , we only need to verify that $\alpha$ satisfies Definition 2.1(ii). To this end let $v \in \alpha \wedge K$ be given. Then $v-\alpha \leq 0$ in $\Omega$ and in view of (6.2) we get

$$
\begin{aligned}
\langle A \alpha- & f, v-\alpha\rangle+\int_{\Omega} j^{o}(\alpha ; v(x)-\alpha) d x \\
& =\int_{\Omega}\left(j^{o}(\alpha ; v(x)-\alpha)-f(x)(v(x)-\alpha)\right) d x \\
& =\int_{\Omega}\left(j^{o}(\alpha ;-1)+f(x)\right)(\alpha-v(x)) d x \geq 0 \quad \forall v \in \alpha \wedge K,
\end{aligned}
$$

which proves that $\alpha$ is a subsolution. In a similar way one can show that under (6.3), the constant $\beta \geq 0$ is a supersolution. Finally, (c) follows immediately from (a) and (b).

In order to apply Theorem 4.1 to our example, we only need to make sure that, in addition, $\beta \wedge K \subset K$ and $\alpha \vee K \subset K$ is satisfied. For the obstacle problem $\beta \wedge K \subset K$ is trivially satisfied and $\alpha \vee K \subset K$ holds provided $\alpha \leq \psi(x)$ for a.e. $x \in \Omega$.

Moreover, straightforward calculations show that both lattice conditions in (5.9) are satisfied for our convex set $K$ here. Thus, Theorem 5.3 also holds in the present example if $\alpha \leq \psi(x)$ for a.e. $x \in \Omega$.

Remark 6.2. Our main goal is a general sub-supersolution approach for variationalhemivariational inequalities and the example given here illustrates the above results in a simple circumstance. Calculations of nonconstant sub-supersolutions in inclusions and variational inequalities were presented, for example, in $[3,4,7]$.

Applications of the sub-supersolution method presented above to some variationalhemivariational inequalities in material science (in which nonconstant sub-supersolutions are constructed) will be studied in a forthcoming project.

6.2. Generalization. Our discussions above could be extended to the case where the principal operator $A$ is perturbed by a lower-order term $G$. The inequality (1.1) is extended to

$$
u \in K:\langle A u+G u-f, v-u\rangle+\int_{\Omega} j^{o}(u ; v-u) d x \geq 0 \quad \forall v \in K
$$

where $G$ is the Nemytskij operator associated with a Carathéodory function $g: \Omega \times \mathbb{R} \times$ $\mathbb{R}^{N} \rightarrow \mathbb{R}:$

$$
\langle G u, v\rangle=\int_{\Omega} g(\cdot, u, \nabla u) v d x \quad \forall u, v \in V
$$


For the integral in (6.7) to be defined, we need some growth condition on $g$, which will be specified later. Note that the operator $A+G$ is not coercive in general. The definition of supersolutions of (6.6) now becomes as follows.

Definition 6.3. A function $\bar{u} \in V$ is called a supersolution of (6.6) if the following holds:

(i) $\bar{u} \geq 0$ on $\partial \Omega$,

(ii) $G \bar{u} \in L^{q}(\Omega)$,

(iii) $\langle A \bar{u}+G \bar{u}-f, v-\bar{u}\rangle+\int_{\Omega} j^{o}(\bar{u} ; v-\bar{u}) d x \geq 0$, for all $v \in \bar{u} \vee K$.

We have a similar definition for subsolutions of (6.6). Combining this notion of subsupersolutions with appropriate modifications of the arguments in Section 5, we can prove the following existence and extremality result for (6.6).

Theorem 6.4. (a) Assume the hypotheses (A1)-(A3), (H), and that (6.6) has subsolutions $\underline{u}_{1}, \ldots, \underline{u}_{k}$ and supersolutions $\bar{u}_{1}, \ldots, \bar{u}_{m}$ such that

$$
\underline{u}:=\max \left\{\underline{u}_{1}, \ldots, \underline{u}_{k}\right\} \leq \bar{u}:=\min \left\{\bar{u}_{1}, \ldots, \bar{u}_{m}\right\},
$$

and $\bar{u}_{i} \wedge K \subset K, \underline{u}_{j} \vee K \subset K$ for all $1 \leq i \leq m, 1 \leq j \leq k$. Suppose furthermore $g$ has the growth condition

$$
|g(x, u, \xi)| \leq k_{2}(x)+c_{6}|\xi|^{p-1}
$$

for a.e. $x \in \Omega$, all $\xi \in \mathbb{R}^{N}$, and all $u \in \mathbb{R}$ such that

$$
\min \left\{\underline{u}_{1}(x), \ldots, \underline{u}_{k}(x)\right\} \leq u \leq \max \left\{\bar{u}_{1}(x), \ldots, \bar{u}_{m}(x)\right\},
$$

where $k_{2} \in L^{q}(\Omega), c_{6}>0$. Then there exists a solution $u$ of (6.6) such that

$$
\underline{u} \leq u \leq \bar{u} .
$$

(b) Furthermore, if $K$ satisfies (5.9), then under the assumptions in (a), (6.6) possesses extremal solutions within $[\underline{u}, \bar{u}]$.

Proof. To prove the assertion in part (a), we follow the idea of the proof of Theorem 4.1. We first note that variational-hemivariational inequality (6.6) is equivalent to the following. Find $u \in V_{0}$ such that

$$
\langle A u+G u-f, v-u\rangle+I_{K}(v)-I_{K}(u)+\int_{\Omega} j^{o}(u ; v-u) d x \geq 0 \quad \forall v \in V_{0},
$$

where $I_{K}$ denotes the indicator function related to $K$. However, unlike in Theorem 4.1 the functions $\underline{u}$ and $\bar{u}$ defined in (6.8) are no longer sub- and supersolutions, respectively. Therefore our existence proof will be based on the following modified auxiliary truncated problem: find $u \in V_{0}$ such that

$$
\langle A u-f+\lambda B(u)+P u, v-u\rangle+I_{K}(v)-I_{K}(u)+\int_{\Omega} j^{o}(u ; v-u) d x \geq 0 \quad \forall v \in V_{0},
$$


where $B$ is the cut-off operator as given by (2.9) and $\lambda \geq 0$ is some free parameter to be specified later. The operator $P: V_{0} \rightarrow V_{0}^{*}$ is defined by

$$
P u:=G \circ T u+\sum_{i=1}^{m}\left|G \circ T^{i} u-G \circ T u\right|-\sum_{j=1}^{k}\left|G \circ T_{j} u-G \circ T u\right|,
$$

where the truncation operators $T_{j}, T^{i}, T: V \rightarrow[\underline{u}, \bar{u}] \subset V$ are defined as follows:

$$
\begin{gathered}
T u(x)= \begin{cases}\underline{u}(x) & \text { if } u(x)<\underline{u}(x), \\
u(x) & \text { if } \underline{u}(x) \leq u(x) \leq \bar{u}(x), \\
\bar{u}(x) & \text { if } u(x)>\bar{u}(x),\end{cases} \\
T_{j} u(x)= \begin{cases}\underline{u}_{j}(x) & \text { if } u(x)<\underline{u}_{j}(x), \\
u(x) & \text { if } \underline{u}_{j}(x) \leq u(x) \leq \bar{u}(x), \\
\bar{u}(x) & \text { if } u(x)>\bar{u}(x),\end{cases} \\
T^{i} u(x)= \begin{cases}\underline{u}(x) & \text { if } u(x)<\underline{u}(x), \\
u(x) & \text { if } \underline{u}(x) \leq u(x) \leq \bar{u}_{i}(x), \\
\bar{u}_{i}(x) & \text { if } u(x)>\bar{u}_{i}(x),\end{cases}
\end{gathered}
$$

for $1 \leq i \leq m, 1 \leq j \leq k, x \in \Omega$. The operators $G \circ T, G \circ T_{j}, G \circ T^{i}$ stand for the compositions of the Nemytskij operator $G$ and the truncation operators $T, T_{j}, T^{i}$, respectively, and we have

$$
\left\langle\left|G \circ T_{j} u-G \circ T u\right|, v\right\rangle=\int_{\Omega}\left|g\left(\cdot, T_{j} u, \nabla T_{j} u\right)-g(\cdot, T u, \nabla T u)\right| v d x
$$

for all $u, v \in V_{0}$. Since $T_{j}, T^{i}, T: V_{0} \rightarrow V_{0}$ are bounded and continuous, it follows in view of the growth condition imposed on $g$ that $P: V_{0} \rightarrow L^{q}(\Omega) \subset V_{0}^{*}$ is bounded and continuous as well. Moreover, by applying [1, Theorem D.2.1] one sees that $A+\lambda B+P$ : $V_{0} \rightarrow V_{0}^{*}$ is continuous, bounded, and pseudomonotone. Introducing the same functional $J$ as in the proof of Theorem 4.1, we can show that the multivalued operator $A+\lambda B+P+\partial\left(\left.J\right|_{V_{0}}\right): V_{0} \rightarrow 2^{V_{0}^{*}}$ is pseudomonotone, bounded, and due to the growth condition on $g$ as well as the mapping properties of the truncation operators, it is also coercive for $\lambda$ chosen sufficiently large. Hence, by similar arguments as in the proof of Theorem 4.1, we infer that (6.13) has a solution $u$. The proof of the existence result of part (a) is accomplished provided any solution $u$ of (6.13) can be shown to satisfy

$$
\underline{u}_{j} \leq u \leq \bar{u}_{i}, \quad 1 \leq i \leq m, 1 \leq j \leq k
$$

This is because then $u$ satisfies also $\underline{u} \leq u \leq \bar{u}$ which finally results in $T u=u, T_{j} u=u$, $T^{i} u=u$, and thus $P u=G u$ as well as $B u=0$ showing that $u$ is a solution of (6.12) (i.e., of (6.6)) within $[\underline{u}, \bar{u}]$. 
We first show that $u \leq \bar{u}_{l}$ for $l \in\{1, \ldots, m\}$ fixed. By Definition 6.3 we have $\bar{u}_{l} \geq 0$ on $\partial \Omega$, and

$$
\left\langle A \bar{u}_{l}+G \bar{u}_{l}-f, v-\bar{u}_{l}\right\rangle+\int_{\Omega} j^{o}\left(\bar{u}_{l} ; v-\bar{u}_{l}\right) d x \geq 0 \quad \forall v \in \bar{u}_{l} \vee K,
$$

and $u$ is a solution of auxiliary problem (6.13) which is equivalent to the following. Find $u \in K$ such that

$$
\langle A u-f+\lambda B(u)+P u, v-u\rangle+\int_{\Omega} j^{o}(u ; v-u) d x \geq 0 \quad \forall v \in K .
$$

We apply the special test function $v=\bar{u}_{l} \vee u=\bar{u}_{l}+\left(u-\bar{u}_{l}\right)^{+}$in (6.18) and $v=\bar{u}_{l} \wedge u=$ $u-\left(u-\bar{u}_{l}\right)^{+}(\in K)$ in (6.19), and get by adding the resulting inequalities the following one:

$$
\begin{aligned}
& \left\langle A \bar{u}_{l}-A u,\left(u-\bar{u}_{l}\right)^{+}\right\rangle+\left\langle\lambda B(u)+P u-G \bar{u}_{l},-\left(u-\bar{u}_{l}\right)^{+}\right\rangle \\
& +\int_{\Omega}\left(j^{o}\left(\bar{u}_{l} ;\left(u-\bar{u}_{l}\right)^{+}\right)+j^{o}\left(u ;-\left(u-\bar{u}_{l}\right)^{+}\right)\right) d x \geq 0,
\end{aligned}
$$

which yields due to

$$
\left\langle A u-A \bar{u}_{l},\left(u-\bar{u}_{l}\right)^{+}\right\rangle \geq 0,
$$

the inequality

$$
\left\langle\lambda B(u)+P u-G \bar{u}_{l},\left(u-\bar{u}_{l}\right)^{+}\right\rangle \leq \int_{\Omega}\left(j^{o}\left(\bar{u}_{l} ;\left(u-\bar{u}_{l}\right)^{+}\right)+j^{o}\left(u ;-\left(u-\bar{u}_{l}\right)^{+}\right)\right) d x .
$$

As in (4.17), for the right-hand side of (6.22) we get the estimate

$$
\int_{\Omega}\left(j^{o}\left(\bar{u}_{l} ;\left(u-\bar{u}_{l}\right)^{+}\right)+j^{o}\left(u ;-\left(u-\bar{u}_{l}\right)^{+}\right)\right) d x \leq \int_{\left\{u>\bar{u}_{l}\right\}} c_{1}\left(u(x)-\bar{u}_{l}(x)\right)^{p} d x .
$$

As for the estimates of the terms on the left-hand side of (6.22) we note that $\bar{u}_{l} \geq \bar{u} \geq \underline{u} \geq$ $\underline{u}_{j}$ which by taking into account the definition of the truncation operators yields

$$
\int_{\left\{u>\bar{u}_{l}\right\}} \sum_{j=1}^{k}\left|G \circ T_{j} u-G \circ T u\right|\left(u-\bar{u}_{l}\right) d x=0,
$$


and the following estimates

$$
\begin{aligned}
& \left\langle B(u),\left(u-\bar{u}_{l}\right)^{+}\right\rangle=\int_{\left\{u>\bar{u}_{l}\right\}}(u-\bar{u})^{p} d x \geq \int_{\left\{u>\bar{u}_{l}\right\}}\left(u-\bar{u}_{l}\right)^{p} d x, \\
\left\langle P u-G \bar{u}_{l},\left(u-\bar{u}_{l}\right)^{+}\right\rangle & \int_{\left\{u>\bar{u}_{l}\right\}}\left(P u-G \bar{u}_{l}\right)\left(u-\bar{u}_{l}\right) d x \\
= & \int_{\left\{u>\bar{u}_{l}\right\}}\left[\left(G \circ T u-G \bar{u}_{l}\right)\left(u-\bar{u}_{l}\right)+\sum_{i=1}^{m}\left|G \circ T^{i} u-G \circ T u\right|\left(u-\bar{u}_{l}\right)\right] d x \\
= & \int_{\left\{u>\bar{u}_{l}\right\}}\left(\left(G \circ T u-G \bar{u}_{l}\right)+\left|G \bar{u}_{l}-G \circ T u\right|\right)\left(u-\bar{u}_{l}\right) d x \\
& +\int_{\left\{u>\bar{u}_{l}\right\}} \sum_{i \neq l}\left|G \circ T^{i} u-G \circ T u\right|\left(u-\bar{u}_{l}\right) d x \geq 0 .
\end{aligned}
$$

Thus from (6.22) we get by means of (6.23), and (6.25),

$$
\left(\lambda-c_{1}\right) \int_{\left\{u>\bar{u}_{l}\right\}}\left(u-\bar{u}_{l}\right)^{p} d x \leq 0 .
$$

By selecting $\lambda$ in addition large enough such that $\lambda-c_{1}>0$, from (6.26) we obtain $u \leq$ $\bar{u}_{l}$. In a similar way one can prove that for any $l \in\{1, \ldots, k\}$ one has also $u \geq \underline{u}_{l}$ which completes the proof of part (a) of the theorem.

In order to prove (b), that is, the existence of extremal solutions in $[\underline{u}, \bar{u}]$, we denote again by $\mathscr{S}$ the set of all solutions of $(6.6)$ within $[\underline{u}, \bar{u}]$. Following the line in the proof of Theorem 5.1, one readily verifies the compactness of $\mathscr{Y}$ in $V_{0}$. Due to lattice condition (5.9) assumed in (b), one observes that any solution $u \in \mathscr{Y}$ is, in particular, a subsolution and a supersolution of (6.6). Therefore, the statement of part (a) implies that $\mathscr{S}$ is a directed set. In just the same way as in Step 2 of the proof of Theorem 5.3, the compactness and directedness of $\mathscr{S}$ yield the existence of extremal elements of $\mathscr{Y}$, which completes the proof of the theorem.

Remark 6.5. The results and methods in this paper can be extended to variationalhemivariational inequalities involving more general quasilinear elliptic operators of Leray-Lions type and functions $j: \Omega \times \mathbb{R} \rightarrow \mathbb{R}$ depending also on the space variable $x$, which, however, has been omitted in order to avoid too many technicalities and in order to emphasize the main ideas.

We could also extend the above results to more general cases where the operator $A$ satisfies a monotonicity condition such as

$$
\left\langle A u_{1}-A u_{2},\left(u_{1}-u_{2}\right)^{+}\right\rangle \geq 0
$$

for $u_{1}, u_{2}$ in some appropriate function space (such as $V_{0}$ or its analogue). This extension would allow us to study problems with weighted or degenerate operators. 


\section{References}

[1] S. Carl and S. Heikkilä, Nonlinear Differential Equations in Ordered Spaces, Chapman \& Hall/CRC Monographs and Surveys in Pure and Applied Mathematics, vol. 111, Chapman \& Hall/CRC, Florida, 2000.

[2] S. Carl and V. K. Le, Enclosure results for quasilinear systems of variational inequalities, J. Differential Equations 199 (2004), no. 1, 77-95.

[3] S. Carl, V. K. Le, and D. Motreanu, The sub-supersolution method and extremal solutions for quasilinear hemivariational inequalities, Differential Integral Equations 17 (2004), no. 1-2, $165-178$.

[4] S. Carl and D. Motreanu, Extremal solutions of quasilinear parabolic inclusions with generalized Clarke's gradient, J. Differential Equations 191 (2003), no. 1, 206-233.

[5] - Quasilinear elliptic inclusions of hemivariational type: extremality and compactness of the solution set, J. Math. Anal. Appl. 286 (2003), no. 1, 147-159.

[6] F. H. Clarke, Optimization and Nonsmooth Analysis, 2nd ed., Classics in Applied Mathematics, vol. 5, SIAM, Pennsylvania, 1990.

[7] V. K. Le, Existence of positive solutions of variational inequalities by a subsolution-supersolution approach, J. Math. Anal. Appl. 252 (2000), no. 1, 65-90.

[8] Sub-supersolutions and the existence of extremal solutions in noncoercive variational inequalities, JIPAM. J. Inequal. Pure Appl. Math. 2 (2001), no. 2, article 20, pp. 1-16.

[9] Subsolution-supersolution method in variational inequalities, Nonlinear Anal. Ser. A: Theory Methods 45 (2001), no. 6, 775-800.

[10] Z. Naniewicz and P. D. Panagiotopoulos, Mathematical Theory of Hemivariational Inequalities and Applications, Monographs and Textbooks in Pure and Applied Mathematics, vol. 188, Marcel Dekker, New York, 1995.

[11] E. Zeidler, Nonlinear Functional Analysis and Its Applications. II/B, Nonlinear Monotone Operators, Springer-Verlag, New York, 1990, translated from German by the author and Leo F. Boron.

S. Carl: Fachbereich Mathematik und Informatik, Institut für Analysis, Martin-Luther-Universität, Halle-Wittenberg, 06099 Halle, Germany

E-mail address: carl@mathematik.uni-halle.de

Vy K. Le: Department of Mathematics and Statistics, University of Missouri-Rolla, Rolla, MO 65401, USA

E-mail address: vy@umr.edu

D. Motreanu: Département de Mathématiques, Université de Perpignan, 52 Avenue Paul Alduy, 66860 Perpignan, France

E-mail address: motreanu@univ-perp.fr 


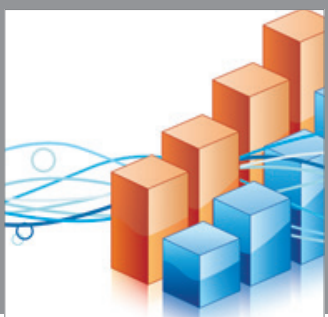

Advances in

Operations Research

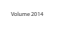

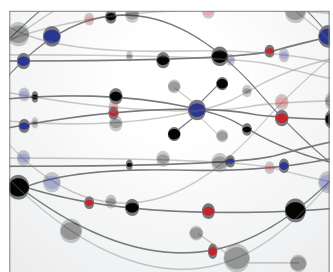

\section{The Scientific} World Journal
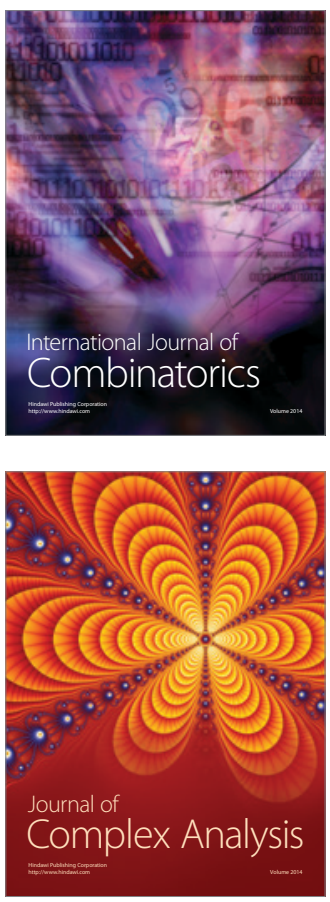

International Journal of

Mathematics and

Mathematical

Sciences
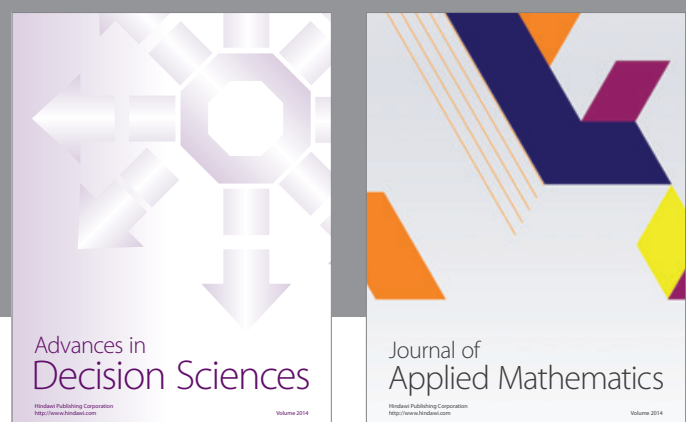

Journal of

Applied Mathematics
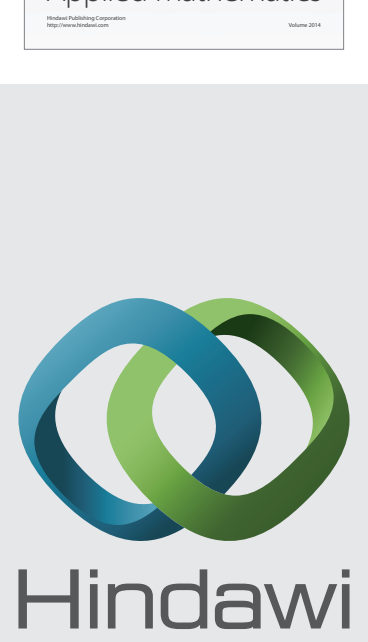

Submit your manuscripts at http://www.hindawi.com
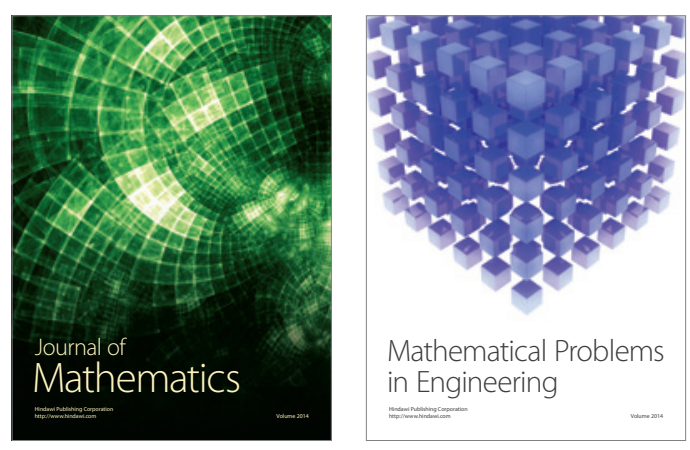

Mathematical Problems in Engineering
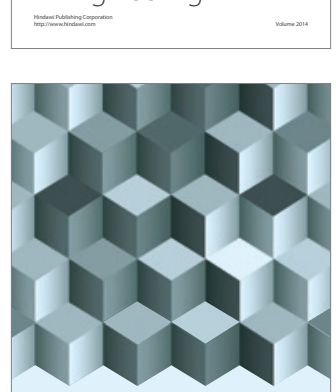

Journal of

Function Spaces
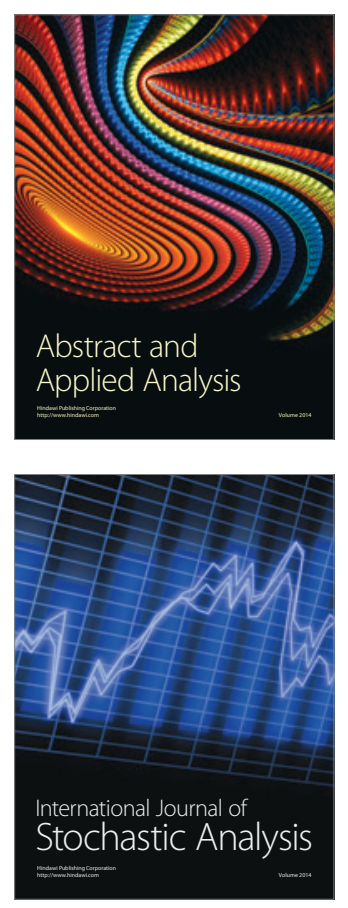

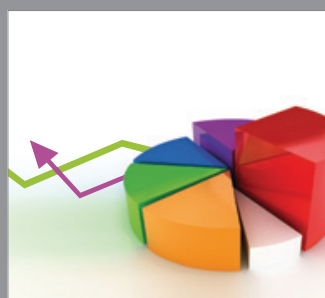

ournal of

Probability and Statistics

Promensencen
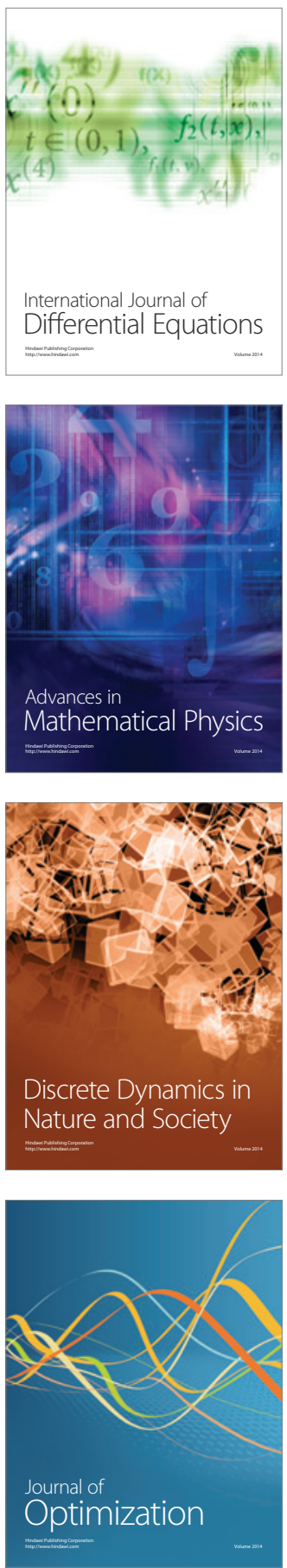